\title{
THE SCATTER AND ABSORPTION OF LIGHT BY THE EXCISED CORNEA AT VARIOUS WAVELENGTHS DETERMINED BY A PHOTOELECTRIC METHOD. THE EFFECT OF TRAUMA AND VACCINIAL INFECTION
}

\author{
By J. MAXWELL LITTLE, JAMES W. MANKIN, CHARLES H. REID, AND \\ GEORGE T. HARRELL
}

(From the Departments of Physiology and Pharmacology, and Internal Medicine, the Bowman Gray School of Medicine of Wake Forest College, Winston-Salem, N. C.)

(Received for publication October 20, 1946)

The effects of immunity and x-ray therapy on the extent of permanent damage to the cornea resulting from the invasion of the cornea by vaccinia have not previously been subjected to experimental study. In preliminary experiments (1) an attempt was made to judge the effects of such treatment by grading the opacities according to an arbitrary scale. It was soon realized that this method was inadequate. In order to obtain a more accurate evaluation of therapy, it was necessary to devise a procedure for estimating objectively the degree of corneal opacity. By utilizing a technique which measures the per cent transmission of light by the cornea in vitro, it was possible to estimate the degree of impairment to vision resulting from diminished light transmission due to opacities. This procedure is described here, and in addition data are given on the light transmission of normal rabbit corneas, corneas subjected to trauma, and corneas inoculated with vaccinia but not treated. The effects of therapy will be described elsewhere (1).

\section{PROCEDURE}

The degree of opacity was estimated by measuring the transmission of light through the cornea in the Evelyn photo-electric micro-colorimeter (2). The intensity of the incident beam of light was adjusted so that the galvanometer read 100 per cent transmission when the light was passed through 0.9 per cent $\mathrm{NaCl}$ solution. Within 15 minutes after the rabbit was sacrificed the cornea was placed in the cell with the convex surface downward. The plunger was inserted, and the per cent transmission of the light was read. The plunger was adjusted to rest lightly on the cornea thus causing it to lie flat. Appropriate filters (red, green and blue ${ }^{1}$ ) were used so that the visual spectrum was covered at the intervals given in

1 The filters used were obtained from the manufacturer of the microcolorimeter and were designated by the wavelength in millimicrons of the maximum light transmitted. The limits of transmission are indicated in Table $I$.
Tables I and II. At least two determinations of transmission were always made on each cornea at each wavelength.

It usually was not necessary to trim the cornea to fit the cell, but on occasion this was necessary. The trimmed edge always showed some opacity resulting from the trauma, but care was taken to exclude the edge from the path of the light beam. After insertion of the plunger the cornea was inspected to exclude the presence of air bubbles and foreign material.

In only a few instances did the opacity cover the entire field. In most instances the opacity was relatively small in comparison with the field, and in these cases the opacity was centered in the field.

Experiments reported here include studies made upon normal rabbit corneas (group Q) ; corneas which had been anesthetized with a 2 per cent butyn sulfate solution, scratched with a needle and treated with $105 \mathrm{r}$ of $\mathrm{x}$-ray (group L) ; untraumatized corneas removed from rabbits in which the other eye had been inoculated with vaccinia (group P) ; and corneas which had been inoculated with vaccinia, but in which no form of therapy was given (group K). Beginning 25 days after the start of the experiment one animal from each group was sacrificed on successive days and the measurements were made. Preliminary experiments (1) had shown that this lapse of time was sufficient for the development of permanent opacities.

\section{RESULTS}

Table I shows that in the normal corneas there was a progressive decrease in the average transmission of light as the filters were changed to allow the transition from long to short wavelengths. This decrease could be due to the absorption of light by the cornea, since Wald (3) states that there is some absorption by the normal cornea in the violet region of the spectrum. It could also be due to the scattering of light by the cornea. Rayleigh's law states that the intensity of the scattered light is inversely proportional to the fourth power of the wavelength; therefore, the scattered radiation would be greatly increased at the shorter wavelengths. The data given in Table I have 
TABLE I

The per cent transmission of light $(T)$ by normal rabbit corneas, Group $Q$, at different wavelengths

\begin{tabular}{|c|c|c|c|c|c|c|c|}
\hline No. & $\begin{array}{c}\text { *F } 660 \mathrm{~m} \mu \\
+(635-720 \mathrm{~m} \mu)\end{array}$ & $\underset{(595-660)}{F 620 \mathrm{m \mu}}$ & $\underset{(515-570)}{\mathrm{F} 540 \mathrm{~m} \mu}$ & $\underset{(495-550)}{\text { F520 m }}$ & $\begin{array}{c}\mathrm{F} 490 \mathrm{~m} \mu \\
(465-530)\end{array}$ & $\begin{array}{l}\text { F440 m } \mu \\
(410-475)\end{array}$ & $\begin{array}{r}\mathrm{F} 420 \mathrm{~m} \mu \\
(380-460)\end{array}$ \\
\hline $\begin{array}{r}1 \\
2 \\
3 \\
4 \\
5 \\
6 \\
7 \\
8 \\
9 \\
10\end{array}$ & $\begin{array}{c}\text { per cent } \\
99.50 \\
99.00 \\
99.00 \\
97.00 \\
99.00 \\
99.25 \\
99.25 \\
99.25 \\
98.75 \\
98.50\end{array}$ & $\begin{array}{c}\text { per cent } \\
99.50 \\
99.00 \\
98.75 \\
96.00\end{array}$ & $\begin{array}{l}\text { per cent } \\
98.00 \\
97.25 \\
97.00 \\
94.25 \\
97.50 \\
97.25 \\
97.25 \\
96.50 \\
97.00 \\
96.50\end{array}$ & $\begin{array}{c}\text { per cent } \\
97.50 \\
97.00 \\
96.00 \\
93.75 \\
96.50 \\
\\
97.00 \\
93.00 \\
96.50 \\
96.00\end{array}$ & $\begin{array}{l}\text { per cent } \\
97.50 \\
96.00 \\
96.00 \\
92.00 \\
\\
96.00 \\
92.50 \\
96.00 \\
95.50\end{array}$ & $\begin{array}{c}\text { per cent } \\
95.00 \\
93.50 \\
93.50 \\
90.00 \\
\\
\\
\\
89.00 \\
93.50 \\
93.00\end{array}$ & $\begin{array}{l}\text { per cent } \\
93.00 \\
91.50 \\
92.00 \\
88.00 \\
92.00 \\
\\
92.00 \\
88.00 \\
92.00 \\
91.00\end{array}$ \\
\hline Mean & 98.9 & 98.1 & 96.9 & 95.4 & 95.2 & 92.5 & 91.0 \\
\hline
\end{tabular}

* Wavelength of maximum transmission.

† Wavelength transmission limits.

been examined from this viewpoint to see if scattering is a factor.

If $T$ represents the per cent of light transmitted by the cornea, then $100-T$ would represent the per cent scattered. According to Rayleigh's law then

$$
(100-T)=k / \lambda^{4} \text {. }
$$

The constant $k$ was calculated for $1 / \lambda^{3}, 1 / \lambda^{4}$, and $1 / \lambda^{5}$, using the observed data for the value of $100-T$ at $\lambda=420 \mathrm{~m} \mu$, and the above formula. With these values for $k$, theoretical lines were constructed to show the anticipated relationship between $100-T$ and the above reciprocal functions. Figure 1 shows that when the observed values for

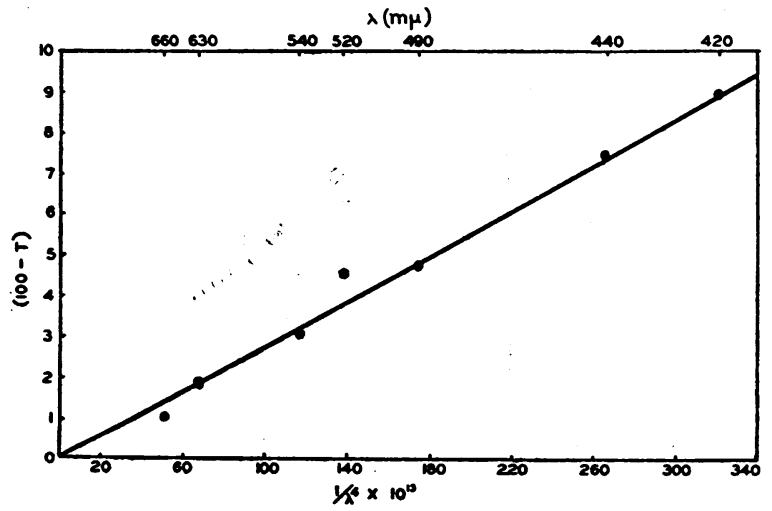

Fig. 1. Relationship between the Observed Values of $(100-T)$, Obtained on Normal Corneas (Group Q), aNd The Theoretical Line Constructed from the EQuation $(100-T)=k / \lambda^{4}$, When $k$, Calculated From the Observed Value of $(100-T)$ at $\lambda=420 \mathrm{~m} \mu$, WAS $28 \times 10^{10}$
$100-T$ were plotted against $1 / \lambda^{4}$ for each wavelength the observed data fell either on or very close to the theoretical line. Using $1 / \lambda^{3}$ the observed values were consistently considerably less than those expected from the theoretical line, and using

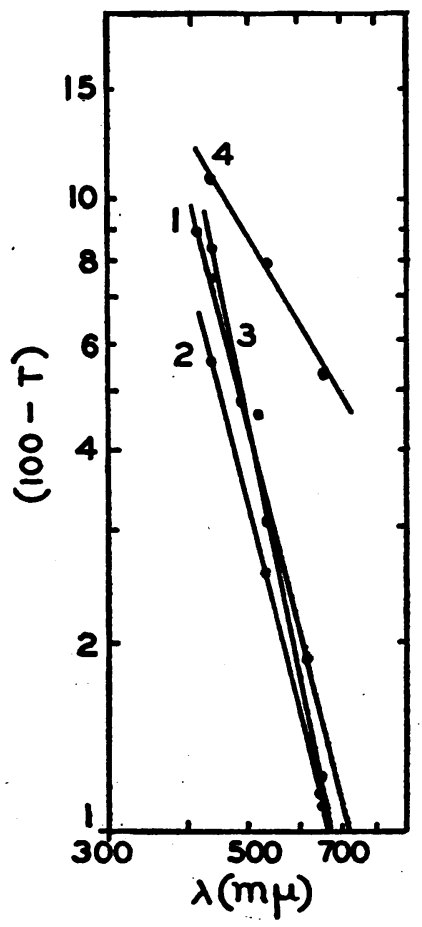

Fig. 2. Relationship between Average Observed ValUES OF $(100-T)$ AND the WaVElength of Light
(1) normal corneas, slope $=-4.0$
(2) Group $P$, slope $=-4.0$
(3) Group L, slope $=-4.7$
(4) Group $K$, slope $=-1.8$ 
$1 / \lambda^{5}$ they were consistently considerably greater than the expected values at all wavelenths greater than $420 \mathrm{~m} \mu$. This is illustrated further by the fact that when the observed values for $100-T$ were plotted against the wavelengths on double logarithmic paper (Figure 2) the slope of the resulting line was minus 4 , indicating that $100-T$ is proportional to $1 / \lambda^{4}$. Thus it appears that the data obtained on normal rabbit corneas obey Rayleigh's law, and that the decrease in transmission at the shorter wavelengths is due to scattering of light rather than to absorption.

The data obtained for the transmission of light by the untraumatized corneas (group P), the corneas which were anesthetized, scratched and treated with x-ray (group L), and the corneas which were inoculated with vaccinia but were untreated (group $\mathrm{K}$ ) are presented in Table II. When the average

TABLE II

The per cent loss of transmission of light by rabbit corneas

Group K: inoculated with vaccinia, untreated; group L: anesthetized, scratched with needle and subjected to x-ray; group P: untraumatized corneas from opposite eye of rabbits with ocular vaccinia

\begin{tabular}{|c|c|c|c|c|c|c|c|c|c|}
\hline \multirow{2}{*}{ Group } & \multicolumn{3}{|c|}{$\begin{array}{l}100-T \\
\text { F660 }\end{array}$} & \multicolumn{3}{|c|}{$\begin{array}{c}100-T \\
\text { F540 }\end{array}$} & \multicolumn{3}{|c|}{$\begin{array}{l}100-T \\
\text { F440 }\end{array}$} \\
\hline & $\mathbf{K}$ & $\mathbf{L}$ & $\mathbf{P}$ & $\mathbf{K}$ & $\mathbf{L}$ & $\mathbf{P}$ & $\mathbf{K}$ & $\mathbf{L}$ & $\mathbf{P}$ \\
\hline $\begin{array}{r}1 \\
2 \\
3 \\
4 \\
5 \\
6 \\
7 \\
8 \\
9 \\
10 \\
11 \\
12 \\
13 \\
14 \\
15 \\
16 \\
17 \\
18 \\
19\end{array}$ & $\begin{array}{r}5.50 \\
2.75 \\
3.75 \\
23.75 \\
7.25 \\
0.50 \\
1.75 \\
3.50 \\
4.25 \\
5.00 \\
3.75 \\
4.25 \\
5.50 \\
3.50 \\
1.00\end{array}$ & $\begin{array}{l}1.75 \\
1.25 \\
0.75 \\
1.75 \\
1.00 \\
1.50 \\
1.50 \\
0.75 \\
1.25 \\
0.75\end{array}$ & $\begin{array}{l}1.25 \\
1.75 \\
1.00 \\
2.50 \\
0.75 \\
0.75 \\
1.00 \\
1.00 \\
0.00 \\
0.50 \\
0.75 \\
1.25 \\
0.50 \\
0.25 \\
2.00 \\
0.00 \\
2.00 \\
1.50 \\
2.75\end{array}$ & $\begin{array}{r}10.25 \\
4.00 \\
6.00 \\
32.00 \\
11.00 \\
3.75 \\
3.25 \\
6.50 \\
6.50 \\
8.00 \\
4.50 \\
6.00 \\
7.50 \\
5.25 \\
2.25\end{array}$ & $\begin{array}{l}4.00 \\
3.00 \\
2.50 \\
3.50 \\
2.50 \\
3.75 \\
3.50 \\
\mathbf{3 . 5 0} \\
\mathbf{2 . 5 0} \\
\mathbf{2 . 7 5}\end{array}$ & $\begin{array}{l}2.50 \\
2.75 \\
2.75 \\
3.50 \\
1.75 \\
2.50 \\
2.50 \\
3.00 \\
2.00 \\
3.00 \\
3.00 \\
3.00 \\
2.50 \\
2.00 \\
3.00 \\
1.75 \\
2.50 \\
2.75 \\
3.50\end{array}$ & $\begin{array}{r}46.00 \\
18.00 \\
8.00 \\
6.00 \\
11.50 \\
10.50 \\
14.50 \\
8.00 \\
10.00 \\
12.00 \\
9.00 \\
6.00\end{array}$ & $\begin{array}{r}6 \\
6.50 \\
7.00 \\
11.25\end{array}$ & $\begin{array}{l}5.50 \\
5.50 \\
5.50 \\
5.00 \\
6.00 \\
5.50 \\
5.50 \\
7.00 \\
4.50 \\
5.00 \\
6.50 \\
6.00\end{array}$ \\
\hline $\begin{array}{l}\text { Mean } \\
\mathbf{\sigma}\end{array}$ & $\begin{array}{c}5.06 \\
15 \\
5.46\end{array}$ & $\begin{array}{c}1.23 \\
10 \\
0.40\end{array}$ & $\begin{array}{c}1.13 \\
19 \\
0.79\end{array}$ & $\begin{array}{l}7.78 \\
15 \\
7.13\end{array}$ & $\begin{array}{c}3.15 \\
10 \\
0.57\end{array}$ & $\begin{array}{l}2.64 \\
19 \\
0.51\end{array}$ & $\begin{array}{c}13.29 \\
12 \\
10.86\end{array}$ & & $\begin{array}{c}5.63 \\
12 \\
0.68\end{array}$ \\
\hline
\end{tabular}

values for $100-T$ in these groups were plotted against the wavelengths, using double logarithmic paper (Figure 2), it was found that the lines for groups $\mathrm{P}$ and $\mathrm{L}$ did not deviate significantly from a slope of minus 4 . The slight deviation for group $L$ is within the limits of experimental error. The slope for group $\mathrm{K}$ (inoculated untreated corneas) however has a value of minus 1.8 , which indicates that some factor in addition to the scatter dimin- ishes the transmission of light when there is an opacity present. In order to get further information about this unknown factor the values obtained on a number of animals in group $K$ were plotted on double logarithmic paper. Figure 3 shows that the slope varied from minus 4.5 for the least degree of opacity to minus 1.6 for the greatest degree of opacity. This variability in slope is primarily due to a greater decrease in transmission at the longer wavelengths with increasing opacity.

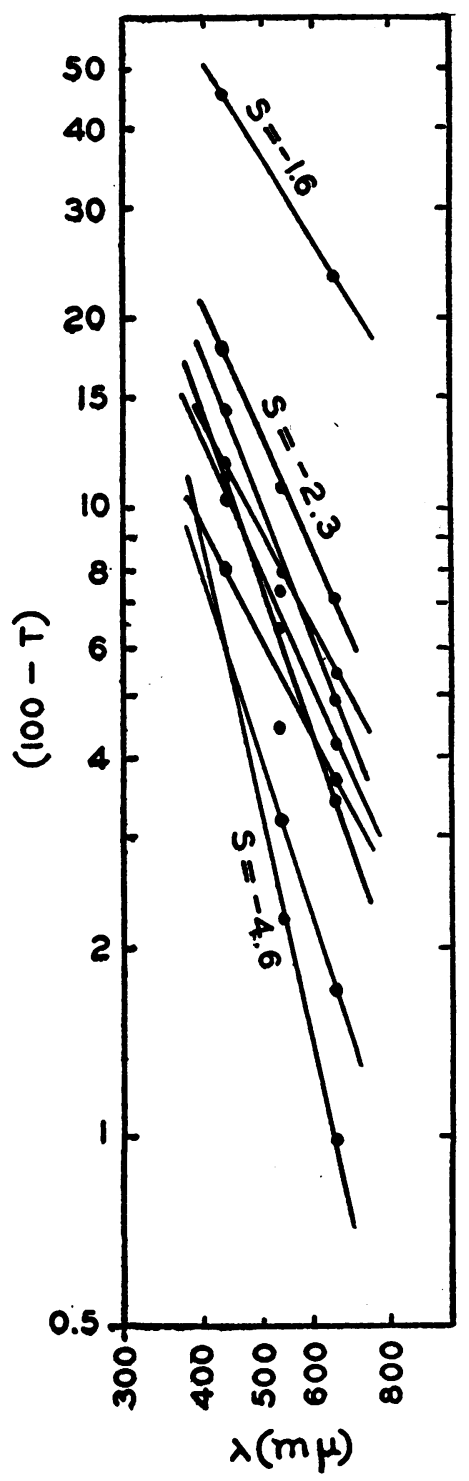

Fig. 3. Relationship between $(100-T)$ and the Wavelength, for 9 of the Corneas from Group K. $s=$ SLOPE 
The significance of the difference between the mean values of $100-T$ (Table II) for groups $L$ and $P$ was tested by "Student's" t-test (4) and was found to be not significant for filter 660 (red). For filter 540 (green), the difference was significant at the 5 per cent level only. The difference between the means for groups $\mathrm{P}$ and $\mathrm{K}$ was significant at the 1 per cent level for filters 660,540 , and 440 (blue).

\section{DISCUSSION}

The procedure described here is a simple objective means for determining the effect of a corneal opacity on the transmission of light at different wavelengths by the cornea. In a few instances no opacity was noticed upon visual inspection of the cornea in situ; however, the transmission of light was impaired in these corneas, and upon careful inspection a rather diffuse opacity was seen.

It is apparent from these experiments that the decrease in transmission of light by the normal cornea at the shorter wavelengths is probably due to a scattering of the incident light by the cornea. This finding raises the question as to whether or not there is any absorption of light by the normal cornea, as reported by Wald (3). It is also apparent that the presence of an opacity introduces some factor, in addition to scatter, which results in a greater defect of transmission. The nature of this factor is unknown, but the data presented in Figure 3 could be explained by the addition of a neutral filter which would absorb equally at all the wavelengths. If this were true the relative effect of the filter would be greatest at the long wavelengths, because at these wavelengths the effect of scatter is- minimal. We would therefore súggest that the effect of an opacity on transmission is due to the equal absorption of light at all wavelengths by the opacity.

The comparison of the means for groups $L$ and $P$ indicates that the trauma associated with anesthesia, scratch, and $\mathrm{x}$-ray produces some perma- nent decrease in the transmission of light by the cornea, even in the absence of evidence of an opacity. The lack of significance in the difference of the means for filter 660 is probably due to the minimal effect of scattering at the long wavelengths together with the factor of sampling variation. It is clear from Figure 2 that trauma without resulting opacity does not alter the nature of the transmission defect found in normal corneas, since the data for group L obey Rayleigh's law.

\section{SUMMARY}

1. The in vitro transmission of light by the rabbit cornea was measured with the Evelyn photoelectric microcolorimeter.

2 . In the normal cornea the per cent transmission is less at the short than at the long wavelengths of light. Since the decrease in transmission is proportional to $1 / \lambda^{4}$ and is consistent with Rayleigh's law, it is concluded that the decrease is due to scattering of the incident light.

3. Trauma to the eye may result in a permanent impairment of light transmission in the absence of an opacity which can be seen by inspection. This decrease is also due probably to scattering of light rather than to absorption.

4. Inoculation of the rabbit cornea with vaccinia results in opacity which causes a significant decrease in the transmission of light. This decrease is probably due to equal absorption of light at all wavelengths.

\section{BIBLIOGRAPHY}

1. Harrell, G. T., Reid, C. H., Little, J. M., Mankin, J. W., Pittman, H. W., Holt, L. B., and Morris, L. M., The effect of roentgen therapy on experimental ocular vaccinia in non-immune and partially immune rabbits. Arch. Ophth. In press.

2. Evelyn, K. A., and Cipriani, A. J., Photoelectric microcolorimeter. J. Biol. Chem., 1937, 117, 365.

3. Wald, G., Human vision and spectrum. Science, 1945, 101, 653.

4. Snedecor, C. W., Statistical Methods. The Iowa State College Press, Ames, Iowa, 1940. 